\title{
RESTRICCIÓN DEL USO DE LA TIERRA EN ÁREAS BAJO AMENAZA VOLCÁNICA: EL CASO DEL ARENAL
}

\author{
Lidier Esquivel Valverde \\ Departamento de Prevención y Mitigación \\ Comisión Nacional de Prevención de Riesgos y Atención de Emergencias \\ Apdo. 5258-1000, San José, Costa Rica, \\ lesquivel@cne.go.cr
}

\begin{abstract}
ANTECEDENTES
El 29 de julio de 1968 se inició la última fase eruptiva del volcán Arenal, evento que dejó como resultado la muerte de 78 personas y pérdida cuantiosas en agricultura, ganadería y vías de comunicación, y que continúa hasta el presente. Melson \& Sáenz (1968) y Sáenz (1977), hacen una detallada descripción del impacto que tuvieron estas erupciones en la región, señalando que fue devastada un área de 15 $\mathrm{km}^{2}$. Los cráteres de impacto alcanzaron Pueblo Nuevo y Tabacón, hasta $5 \mathrm{~km}$ de distancia del cráter. A lo largo de 35 años de actividad, se distinguen algunas erupciones que, por su intensidad, han generado alarma en una población que pareciera, paulatinamente se ha acostumbrado a convivir con esta situación. A pesar de esto, a partir de 1990, el área del Arenal se transforma en una de las principales atracciones turísticas que ofrece el país.

En mayo de 1998, ante la ocurrencia de flujos piroclásticos, fue necesario tomar medidas
\end{abstract}

para controlar el ingreso de turistas a las áreas de mayor peligro; se cerró el tránsito por la carretera que circunda el volcán entre los costados NESW (ruta 142 La Fortuna - La Palma - Tabacón), cierre del Área de Conservación Arenal (ACA), activación de los comités de emergencia de la región, y establecimiento de alerta roja y amarilla para los sectores afectados por flujos piroclásticos (datos del Centro de Información y Análisis de la CNE).

Otro episodio importante ocurrió el 23 de agosto del 2000, cuando flujos piroclásticos se orientaron hacia el flanco norte del volcán, y perecieron dos personas (un guía costarricense y una niña estadounidense) que estaban ubicados en un lugar conocido como Laguna Cedeño, donde usualmente y sobre todo fines semana, la concentración de personas era sumamente importante. Esto, motivado por la construcción de instalaciones para acampar, canopy, navegación en la laguna, sitios para almorzar y hasta una capilla para eventos religiosos. Un elemento circunstancial que favoreció que el número de 
víctimas no fuera mayor, fue el hecho de que el fenómeno ocurrió en un día laboral y en una temporada turística baja.

\section{VULNERABILIDAD}

No es el objetivo del presente trabajo desarrollar una investigación con el fin de descifrar el nivel de vulnerabilidad de la región; sin embargo, es importante para tener una idea de la condición de esta variable, comentar brevemente tres aspectos que marcan una tendencia.

\section{Aumento de la población}

Según datos del Instituto Nacional de Estadística y Censos, la población en el distrito de La Fortuna ha aumentado entre 1969 y el 2000, en un $111 \%$, aunque no ha sido lineal (Fig. 1).

En este periodo se han presentado dos descensos importantes de la población. El primero en 1973, coincidente con el fuerte sismo que afectó la región el 13 de abril (M 6,5; Plafker, 1973), en el cual perdieron la vida 23 personas sepultadas por deslizamientos, y enormes daños en agricultura, la principal fuente de ingresos de la población en esa época. El siguiente descenso en la curva de población se registró en 1979, coincidiendo con una fuerte crisis económica que afectó severamente todos los sectores del país y, probablemente, potenció un aumento de la migración de esta región hacia otras áreas.

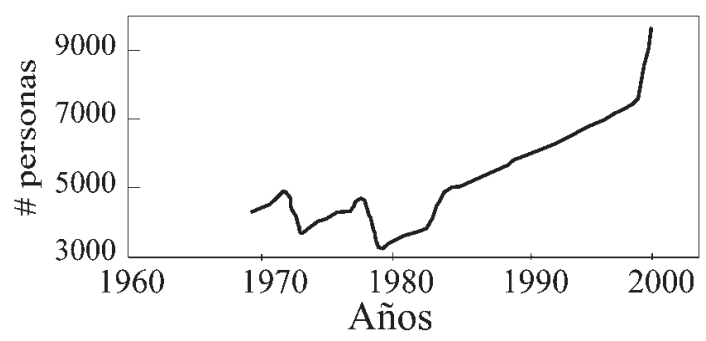

Fig. 1: Crecimiento de la población de la Fortuna.
Igualmente es notorio un cambio en la pendiente del gráfico que muestra el aumento de la población, a partir de inicios de la década de 1990, que coincide claramente con el auge de la inversión turística alrededor del volcán, que ha marcado un cambio rápido e importante en la principal actividad económica de la región, y que trae consigo un impacto social y económico aún no evaluado.

\section{Infraestructura turística}

Como es de esperar, el aumento de turistas en los alrededores del volcán Arenal, también genera una mayor cantidad y diversidad de proyectos turísticos, muchos de los cuales se han instalado cercanos a la población de La Fortuna, pero el polo de desarrollo en este campo, tiene una clara tendencia alrededor del cono volcánico, a lo largo de la principal vía de comunicación, la ruta 142 (La Fortuna - La Palma - Tabacón), en una línea que circunda el volcán en sus flancos este a noroeste. Algunos tramos se encuentran atravesando sectores que han sido identificados con una amenaza importante, inclusive con registros recientes de daños, ligados a los procesos volcánicos anteriormente mencionados.

\section{Manejo de la información}

La información a la población, tanto residentes como turistas, es uno de los aspectos más deficientes que pueden ser encontrados. El Estado y las empresas privadas no han desarrollado campañas agresivas en este sentido. Algunas causas de esta situación son que no se han destinado recursos económicos para ello y que existe el prejuicio entre algunos empresarios de que, al dar información sobre las amenazas y medidas de seguridad, puede afectarse la buena imagen de la región.

En el año 2000, en un curso de Gestión del Riesgo impartido por la Escuela de Geología de la Universidad de Costa Rica, se realizó una encuesta durante un fin de semana en los alrededores del volcán Arenal. Entre los resultados más 
importantes, destaca el desconocimiento total y generalizado (residentes jóvenes, empresarios y turistas), de tres aspectos que son fundamentales: 1) el nivel de actividad de este volcán, 2) las áreas de mayor riesgo y 3) las medidas de seguridad que deben asumir en caso de una erupción.

\section{ASPECTOS LEGALES Y NORMATIVOS}

Aunque sin duda es un factor de vulnerabilidad importante, se considera en un capítulo aparte el tema de la normativa, debido a la relevancia de este instrumento, para el objetivo planteado en este documento. En Costa Rica existe una gran cantidad de instrumentos de carácter legal que regulan diferentes modalidades del ordenamiento territorial. Sin embargo, falta un marco integrador en este tema claramente establecido dentro de la política del estado, de tal forma que fácilmente se pueden encontrar dentro de las normas y regulaciones existentes, duplicidades, conflictos, traslapes y, lo que es peor, vacíos.

En total se identifican al menos 23 instituciones públicas que tienen competencia en el tema, incluyendo los gobiernos locales (81 en total) e instituciones de carácter nacional como la Comisión Nacional de Prevención de Riesgos y Atención de Emergencias (CNE) y el Instituto Nacional de Urbanismo (INVU). Este último cuenta con un área creada exclusivamente para promover el desarrollo e implementación de planes reguladores.

A pesar de esta dispersión, vacíos y hasta cierto punto contradicciones, desarrollar herramientas para el uso de la tierra, y en este caso, con criterio de amenaza (volcánica), sigue siendo una buena opción, para promover acciones de prevención a corto y mediano plazo.

Dada esta situación, y por la premura de tomar medidas concretas, se acordó utilizar el marco legal de la CNE (Ley de Emergencias, 7914), y que entre otros aspectos relacionados con la prevención y mitigación, otorga la potestad para que bajo condiciones de "riesgo inminente de emergencia" (tomado textualmente de la ley 7914), se puedan establecer restricciones en zonas que por su condición de amenaza, así lo requieran.

\section{PROPUESTA DE ZONIFICACION}

Para el aporte de los elementos técnicos necesarios, la CNE, cuenta con una estructura denominada Comités Asesores Técnicos (CAT), los cuales tienen como objetivo principal, asesorar a la institución con el fin de definir políticas sobre diferentes aspectos de la gestión del riesgo.

Uno de estos CAT es el encargado del tema de la vulcanología en nuestro país, y está conformado por los tres centros de investigación que generan información y conocimiento sobre la vigilancia y la amenaza volcánica: La Sección de Sismología, Vulcanología y Exploración Geofísica de la Universidad de Costa Rica, el Área de Amenaza y Auscultación Sísmica y Volcánica del Instituto Costarricense de Electricidad (en conjunto, estos dos conforman la Red Sismológica Nacional, RSN: ICEUCR), y el Observatorio Vulcanológico y Sismológico de Costa Rica (OVSICORI), de la Universidad Nacional.

Luego de los flujos piroclásticos del 23 de agosto del 2000, este grupo técnico, bajo la coordinación de la CNE, recibió la solicitud de la Junta Directiva de esta institución, para abocarse al desarrollo de una propuesta de utilización de las áreas de mayor amenaza en el entorno de la zona de estudio, tomando en cuenta no solo las condiciones de riesgo, sino también reconociendo que hay usos de la tierra históricos, que se dan en la región desde antes que se iniciara el actual periodo eruptivo.

Para el desarrollo de la propuesta no solo se tomó en cuenta la reciente actividad eruptiva que da inicio en 1968, sino que aprovecha la rica información científica que ha sido escrita alrededor de este volcán, y que ha permitido construir una radiografía de los 7000 años de vida (Soto et al., 1998) de uno de los volcanes más activos de América.

La propuesta fue presentada y desarrollada por los vulcanólogos y geólogos Eduardo Malavassi (OVSICORI), Guillermo Alvarado (ICE), Sergio Paniagua (UCR) y Lidier Esquivel (CNE), y publicada en La Gaceta $\mathrm{N}^{\circ} 8$ del 11 de enero del 2001. 


\section{RESTRICCIONES PARA EL USO DEL SUELO EN LOS ALREDEDORES DEL VOLCÁN ARENAL}

La zonificación establecida se basa en dos niveles de restricción con relación al uso propuesto, y son un reflejo de los procesos volcánicos que se espera afecten el entorno en el futuro. La figura 2 muestra las diferentes zonas.

\section{Primer nivel de restricción: Círculo de 5,5 km (uso condicionado)}

El primer nivel corresponde con un círculo de radio $5,5 \mathrm{~km}$ alrededor del cráter del volcán, medido en forma horizontal. El tamaño de este círculo se ha determinado tanto a partir del registro geológico, como de la historia eruptiva reciente, lo que ha permitido identificar que los flujos piroclásticos y sus efectos secundarios, han alcanzado una distancia máxima de 5 km, a partir del cráter. También se consideró que la mayor distancia horizontal de los clastos eyectados en la erupción de 1968, y que dejaron como evidencia una gran cantidad de cráteres de impacto, fue de $5 \mathrm{~km}$. Además, se incluyen dentro de esta área, los cauces activos o intermitentes, que tengan su naciente en el macizo volcánico, hasta su desembocadura en un cauce principal, en este caso, la mayoría, si no todos, son afluentes del río Arenal.

Dentro de esta área circular, cualquier nuevo desarrollo que se pretenda realizar, deberá contar con una investigación de las condiciones locales del terreno donde se quiera construir la obra, que permita determinar ventajas comparativas de tipo topográfico, secuencia estratigráfica del lugar, ubicación respecto a la dirección imperante de los vientos y otros. Dicha investigación deberá ser refrendada por cualquiera de los centros de investigación volcánica del país y conocida por la propia CNE.

Existen tres objetivos que se pretenden con la realización de estas evaluaciones puntuales de amenaza: 1) dar elementos de seguridad y respaldo a los empresarios turísticos, 2) hacer conciencia en ellos mismos de las características del entorno y 3) crear una base de información que permita a futuro contar con parámetros técnico-científicos que nos dé una mayor precisión de las características eruptivas de la región.

\section{Segundo nivel de restricción: No construcción y restricción de la actividad humana}

Este nivel de restricción es mucho más complejo. Evidencia y acepta usos de la tierra históricos en áreas de riesgo, pero que por sus características, no representan una contradicción inmanejable entre la amenaza y las actividades que se realizan en ellos. Además, permite impulsar actividades que no dan cabida a otras que podrían exponer al peligro volcánico mayores cantidades de personas y bienes.

Se reconocen dentro de esta área actividades que manejadas de forma adecuada, tanto en términos de expansión futura, como cantidad y concentración de personas involucradas, pueden continuar, de tal forma que el impacto económico y social sea menor al esperado. Los usos derivados de estas actividades principalmente son ganadería, extracción de materiales no metálicos, conservación de bosque, turismo en pequeña escala, habitación en muy baja densidad y otros.

En la actualidad existen dos desarrollos turísticos importantes dentro de esta área, y que por su tamaño y ubicación respecto a las áreas de influencia de flujos piroclásticos, son totalmente incompatibles con esta amenaza. Sin embargo, la falta de normativa clara y precisa, facilitó las condiciones legales para que no solo se construyeran, sino que se consolidaran dentro de la oferta de distracción que se ofrece en dicha región. Es sabido además, que por el tipo de mecanismo eruptivo, las tecnologías desarrolladas para su detección, las condiciones climáticas imperantes en la región durante gran parte del año, se hace inviable concebir un plan de vigilancia y alerta suficientemente eficaz y eficiente para las personas que laboran y visi$\tan$ sitios bajo estas condiciones.

Se propone en primer lugar y para toda el área mencionada, la prohibición para la construcción de nuevas actividades turísticas (alojamientos, 
restaurantes, balnearios, senderos, campamentos, canopy tours, práctica de senderismo, cabalgatas, construcción de caminos, instalaciones complementarias para prestar servicios turísticos como bodegas, escampaderos y otros) o bien ampliaciones de las ya existentes. Esta recomendación aplica para toda la zona marcada de restricción absoluta, excepto para aquellos sectores que indiquen lo contrario. También se recomienda evitar el establecimiento de nuevas viviendas dentro de las fincas existentes o en segregaciones de ellas.

Además se han definido cuatro subáreas, de acuerdo con su nivel de amenaza (Fig. 2). En orden descendente son las siguientes:

\section{Zona de Restricción 1 (R1)}

Comprende la mayor parte de esta área, y se extiende principalmente en el sector central, en los terrenos aledaños al cráter activo, conformado principalmente por las laderas empinadas del cono volcánico, y además por aquellos sectores alejados del cono, inclusive planos, pero que por razones topográficas y otros, tienen una altísima probabilidad de ser afectados de forma directa en un corto plazo (años o menos), por un flujo piroclástico.

El uso más frecuente es el de bosque en régimen de conservación, y únicamente se han identificado dos desarrollos turísticos, que parcialmente tienen actividades dentro de sus límites.

Se deberán tomar medidas drásticas para que en el menor tiempo posible, no se permitan actividades turísticas que impliquen concentraciones de personas, y que por su uso actual están en conflicto con el nivel de riesgo ampliamente comprobado.

\section{Zona de Restricción 2 (R2)}

Se extiende en una franja hacia el extremo oeste del área de restricción, al pie de una de las laderas del volcán. La estructura más importante dentro de esta zona es la recepción del Área de Conservación Arenal (ACA) y senderos propiedad de esta misma organización. Este sector podrá ser utilizado por la Administración del ACA, de acuerdo con lo que se establece en el apartado Restricciones a la Visita y Tránsito de Personas.

Será imprescindible contar con un Plan de Emergencia debidamente aprobado, demarcación de senderos, salidas de emergencia, e información sobre las amenazas a los visitantes, en varios idiomas. En este sector no se deberá construir nueva infraestructura o servicios a los turistas, a menos que sean senderos, rotulación u otras facilidades al aire libre, que no impliquen concentración de población.

\section{Zona de Restricción 3 (R3)}

Es la franja más al oeste del área en mención, y limita con el embalse del Arenal. Su uso más frecuente es en ganadería y conservación de bosque, auque no existen proyectos turísticos. Por su cercanía a sitios de interés, tienen un potencial de desarrollo muy fuerte. Sin embargo, la dirección predominante de los vientos en la región favorece la caída de cenizas, bloques de lava (1968) y otros productos eruptivos destructivos.

Se recomienda únicamente el establecimiento de senderos para la práctica de caminatas o cabalgatas, así como la pesca en la laguna, de conformidad con las regulaciones existentes en esa materia, o bien otras actividades al aire libre, siempre y cuando no impliquen construcciones de ningún tipo, las cuales deberán estar ubicadas fuera del área de restricción delimitada. Tampoco se permitirá el establecimiento de facilidades para acampar o cualquier actividad que requiera de ello.

\section{Zona de Restricción 4 (R4)}

Se extiende en dos sectores: un área relativamente pequeña hacia el noroeste del sector, que colinda con la carretera El Castillo - Tabacón y otro sector de mayor extensión hacia el extremo este, y que colinda con la carretera La Fortuna - La Palma. El uso más frecuente es en ganadería, protección de bosques, extracción de materiales para la construcción en algunas pequeñas quebradas, y viviendas muy dispersas.

Se recomiendan actividades controladas de índole forestal, agropecuaria y explotación de 


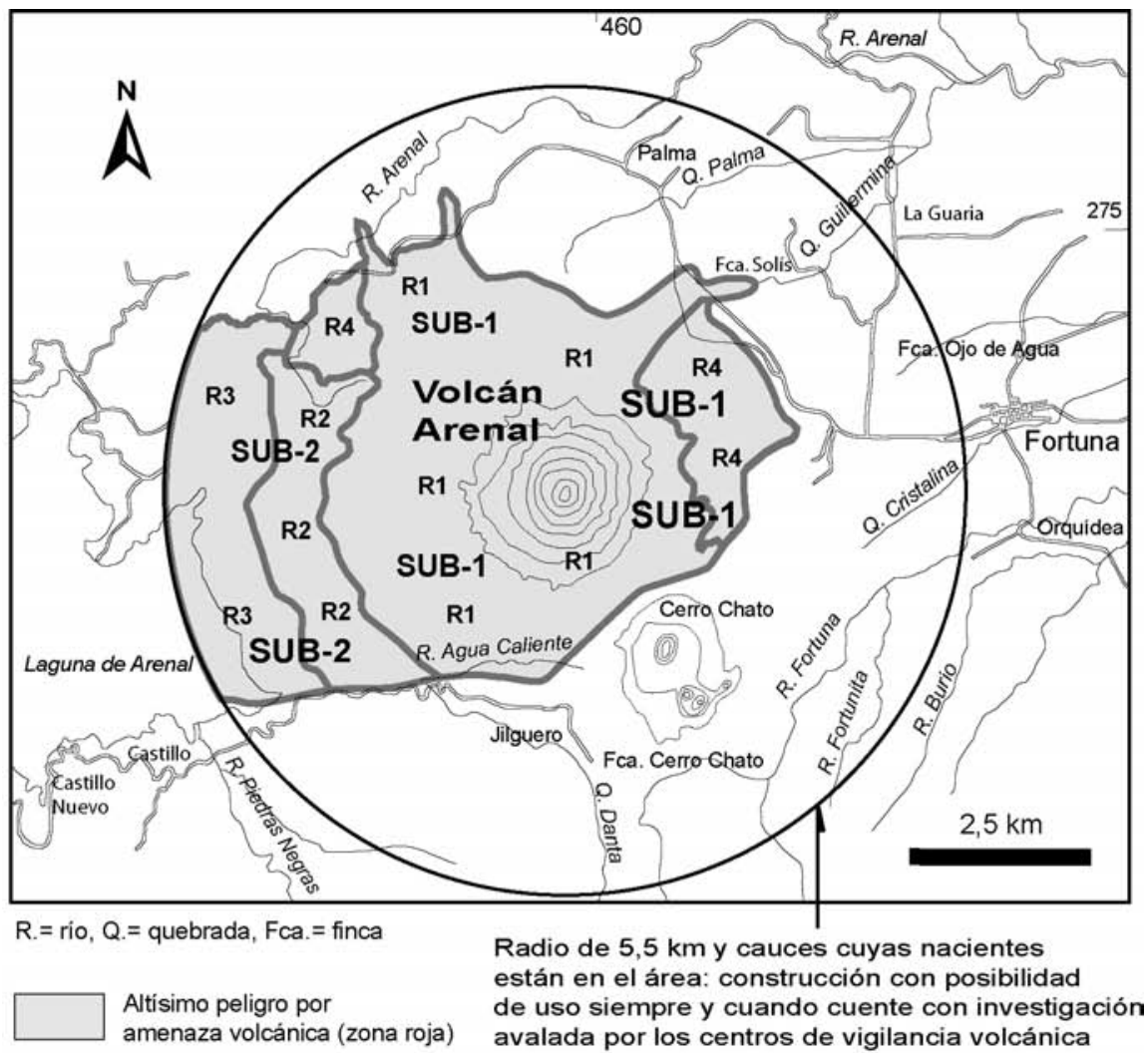

\section{ZONAS DE RESTRICCIÓN PARA EL USO DEL SUELO}

Restricciones para toda la zona roja: No se permiten nuevas actividades turisticas o viviendas

Zona Restricción R1:

Antecedentes históricos y prehistóricos de flujos piroclásticos y nubes ardientes. Prohibición de actividades turisticas

Zona restricción $\mathrm{R} 2$ :

Bajo administración del Área de Conservación Arenal (ACA). Se deberá contar con facilidades para el control de visitantes y suplir información

\section{Zona restricción $\mathrm{R} 3$ :}

Se permite senderismo, cabalgatas, pesca u otras actividades al aire libre. No debe darse nuevas construcciones, ni facilidades para acampar

\section{Zona Restricción R4:}

Se permiten actividades de indole forestal, agropecuaria y explotación de materiales para construcción (tajos) actualmente en funcionamiento. No se permiten ampliaciones de la infraestructura utlizada para estos fines
SUB-1

Subzona de prohibición absoluta de la visita y libre tránsito de personas Se prohibe la visita y libre tránsito de personas, solo en casos calificados.

Restricciones para la visitación y libre tránsito

\section{SUB-2}

Subzona de restricción parcial de visita y libre tránsito de personas. Solo se permite el acceso al flanco oeste del volcán y los terrenos ubicados al oeste del mismo, incluye el área de Conservación Arenal y Laguna del Arenal, la carretera La Fortuna - El Castillo es de libre circulación, con flujo vehicular controlado

Fig. 2: Zonas de restricción de uso del suelo en el volcán Arenal. 
materiales para construcción (tajos), cuando hayan sido establecidas con anterioridad a la declaratoria (11 de enero del 2001). Igualmente, ninguno de los usos anteriores deben implicar concentración de población superior a las siete (7) personas en sitios de alta vulnerabilidad.

No se debe permitir la construcción de nuevas lecherías, establos o bodegas o la ampliación de las preexistentes. Las instalaciones para servicios forestales, agropecuarios y explotación de materiales existentes (siempre que cuenten con los permisos respectivos), podrán ser reparadas.

\section{Restricciones para la visita y tránsito de per- sonas}

Como una medida necesaria para la protección de la vida, tanto de las personas que desarrollan sus actividades diarias en los alrededores, como de los turistas, y mientras el volcán Arenal se mantenga activo, el área restringida será dividida en dos subzonas, en lo que se refiere a la visita de personas y tránsito (Fig. 2).

\section{Subzona de prohibición absoluta de la visita y trán- sito de personas (Sub-1)}

Abarca la totalidad de las zonas demarcadas como R1 y parte de la zona R4. En esta se debe prohibir la visita y el libre tránsito de personas mientras exista la amenaza volcánica, y solo se permitirá el acceso a aquellas personas que cumplan con los siguientes requisitos:

a. Investigadores en Vulcanología al servicio de instituciones nacionales públicas o privadas o de científicos extranjeros acompañados de personal de instituciones nacionales, siempre y cuando porten identificaciones que los acrediten como tales.

b. Funcionarios de instituciones de atención de emergencias realizando operaciones de salvamento o rescate o bien de vigilancia y seguimiento, siempre y cuando porten identificaciones que los acrediten como tales. c. Autoridades de policía en labores de captura de personas o de investigación judicial siempre y cuando porten las identificaciones correspondientes.

\section{Subzona de restricción parcial de la visita y libre tránsito de personas (Sub-2)}

Comprende todas las áreas identificadas como R2 y R3 y se reconoce que aunque existe una amenaza latente, bajo condiciones de relativa normalidad, es posible su permanencia, siempre y cuando se consideren varias regulaciones, correspondientes con su uso. Solo se permitirá la visita de la porción inferior del flanco oeste del volcán y los terrenos ubicados entre el Área de Conservación Arenal y la Laguna del Arenal y el río Arenal, bajo las siguientes condiciones:

a. Se permitirá el ingreso controlado de pequeños grupos de personas (10) al Área de Conservación Arenal (ACA), por tiempo limitado y bajo el estricto control de los funcionarios del ACA, hasta el sector delimitado por la cota de los $600 \mathrm{~m}$ s.n.m., límite que deberá ser debidamente señalado por los funcionarios de esta institución.

b. El ACA debe poseer un sistema de alarma para ordenar evacuaciones, planes de evacuación debidamente aprobados por la $\mathrm{CNE}$, y que las personas no pernocten o acampen dentro de la misma. El único servicio turístico que puede darse dentro de esta zona, es el de guía turístico de grupos de personas, así como el transporte hacia y fuera de la zona. Estos guías deberán ser debidamente certificados por el Instituto Costarricense de Turismo (ICT).

c. La carretera que atraviesa el pie del flanco oeste del volcán será de circulación libre, sin embargo, se recomienda establecer un flujo vehicular controlado, así como la instalación de vallas informativas sobre las diferentes restricciones que existen en la zona.

d. Los terrenos ubicados entre la carretera y el embalse del Arenal y el Río Arenal, podrán ser visitados para realizar actividades 
señaladas para la Zona de Restricción R3, mencionado anteriormente.

\section{CONCLUSIONES}

La actividad volcánica actual, es solo un reflejo de eventos de mucha mayor intensidad que han sido capaces en épocas geológicamente recientes, de devastar áreas alrededor del cono volcánico. El fenómeno de la escasa memoria colectiva característica de nuestros pueblos, se hace latente en esta región del país, donde las reacciones de la población y los empresarios a la posibilidad de regular el uso del suelo, no han sido en su mayoría positivas.

La pérdida de vidas a consecuencia de los flujos piroclásticos en agosto del 2000, permitió retomar un tema muchas veces propuesto, e igual cantidad de veces pospuesto: el de establecer controles de uso de la tierra y acceso de personas en sus alrededores.

Por otro lado, se dieron las condiciones necesarias para que un grupo de expertos de diferentes instituciones, que conforman el Comité Asesor Técnico en Vulcanología, y con el respaldo del sector político-decisorio de la CNE, se abocaran a la discusión de dicha propuesta, la cual fue llevada a los más altos niveles del gobierno central.

La zonificación implementada está muy distante de ser un plan de ordenamiento territorial, sin embargo es un primer paso en la propuesta de un plan de desarrollo para esta región.

Un aspecto deficitario en el proceso desarrollado fue la exclusión del gobierno local, y de las instituciones responsables del ordenamiento territorial en Costa Rica. Aunque se pueden identificar muchas razones de esta exclusión, no se deben tomar como excusa, pues sin duda, la sostenibilidad de las regulaciones planteadas, depende en gran medida de que sean aceptadas de forma generalizada. Algunas circunstancias que se presentaron que favorecieron esta situación, fueron:

a. El poco o ningún conocimiento en el nivel local de aspectos vulcanológicos, y la falta de espacios de discusión ha creado una barrera entre estudiosos del tema y los tomadores de decisión locales y regionales.

b. La fuerte presión que se ejerce en el entorno de la municipalidad (gobierno local), por parte de empresarios locales y de otros ámbitos, para que otorgue permisos de funcionamiento de desarrollo turísticos, ha generado que esta institución no asuma directamente la responsabilidad que le compete en este tema, dejando a un plano nacional dicha decisión, creándose una situación conveniente para el plano local, aduciendo que únicamente acatan las directrices procedentes del Poder Ejecutivo central.

c. La instancia nacional que debe encargarse del ordenamiento territorial, aunque ha desarrollado planes de regulación anteriormente, no cuenta con una política sostenida en materia de gestión de riesgo. Por otro lado, más de la mitad de los cantones del país no cuentan con Plan Regulador, por lo que existen aún vastas extensiones donde debe desarrollarse este instrumento.

d. La premura con que se ha diseñado e implementado la zonificación dificultó la inclusión de otros actores en las discusiones.

Otro aspecto deficiente, es la no participación de los empresarios y la comunidad afectada, siendo esto quizá el aspecto más vulnerable de todo el proceso, sobre todo porque en este momento son los principales detractores de las medidas tomadas, principalmente los primeros, quienes han visto lesionados sus intereses.

Queda por delante, sin embargo una tarea que debe ser permanente en la región, que es la implementación de un proceso de divulgación e información, que permita dar a conocer tanto a los empresarios como a la comunidad en general, los alcances y objetivos de este tipo de regulaciones, necesarias si se quiere alcanzar un desarrollo equitativo con una amenaza volcánica real, en donde el principal atractivo de la región, se puede convertir 
súbitamente en su mayor enemigo, de tal forma que bajo cualquier circunstancia la región pueda ofrecer un servicio turístico seguro.

La mayor dificultad práctica, es el futuro de los dos proyectos turísticos, que se encuentran en este momento en la zona R1, los cuales han contado desde sus inicios con todos los permisos requeridos de funcionamiento, permisos que en todo caso no podían ser negados por no existir una normativa específica para este sitio y para estas situaciones en particular. Queda la tarea legal y moral, de definir su futuro, sin despegarse de nuestra realidad social y económica en una región que apuesta cada vez más al desarrollo turístico, como una forma de vida que sustituya las actividades tradicionales como la agricultura y la ganadería, actualmente en una profunda crisis, que parece no solucionable a un plazo razonable.

La zonificación ha tenido políticamente un costo a corto plazo que parece ser negativo. Sin embargo, a largo plazo el esfuerzo pionero, deberá ser reconocido y enriquecido con una mayor experiencia y conocimiento.

Lamentablemente ese balance negativo que se menciona al corto plazo, pareciera hacer por el momento poco viable poder incorporar una metodología similar, y por supuesto mejorada, como una política de estado necesaria para intervenir otras zonas actualmente bajo condiciones de amenaza (volcánica, sísmica, inundaciones, deslizamientos o tecnológica), y que no cuentan con ningún tipo de herramienta para contener un creciente desarrollo urbano o de otra índole, con un mínimo criterio de gestión del riesgo.

\section{AGRADECIMIENTOS}

El documento "Restricciones para el Uso del Suelo en los Alrededores del volcán Arenal" se publicó en la Gaceta del 11 de enero del año 2001. Por primera vez en el país, se da un producto de este tipo, que adquiere carácter de obligatoriedad para todas las instituciones públicas. $\mathrm{Su}$ autoría surge del trabajo coordinado y en equipo de cuatro instituciones, tres de ellas de investigación en el campo de la amenaza volcánica. Quiero externar mi profundo agradecimiento a los coautores de este instrumento, Guillermo Alvarado, Eduardo Malavassi y Sergio Paniagua, por demostrar que es posible, aun cuando existan diferencias, trabajar por un objetivo común, proteger y ayudar a una comunidad en riesgo. Se agradece, por otra parte, a Gerardo Soto por la edición del texto para su publicación.

\section{REFERENCIAS}

MELSON, W.G. \& SÁENZ, R., 1968: The 1968 eruption of Volcán Arenal: preliminary summary of field and laboratory studies. - Smithsonian Center for Short-Lived Phenomena, Report 7/1968: 1-35.

SÁENZ, R., 1977: Erupción del volcán Arenal en el año 1968. - Rev. Geogr. Amér. Central, 5-6: 149-188.

PLAFKER, G., 1973: Field reconnaissance of the effects of the earthquakes of April 13, 1973, near Laguna de Arenal, Costa Rica. - Bull. Seism. Soc. Am. 63: 1847-1856.

SOTO, G.J., ALVARADO, G.E. \& GHIGLIOTTI, M., 1998: El registro eruptivo del Arenal en el lapso 3000-7000 años antes del presente y nuevas deducciones sobre la edad del volcán. - Bol- OSIVAM, 9(17-18):19-49, San José. 